\title{
Bayesian Uncertainty Quantification for Geomechanical Models at Micro and Macro Scales
}

\author{
Hongyang Cheng ${ }^{1(凶)}$, Vanessa Magnanimo ${ }^{1}$, Takayuki Shuku ${ }^{2}$, \\ Stefan Luding ${ }^{1}$, and Thomas Weinhart ${ }^{1}$ \\ ${ }^{1}$ Faculty of Engineering Technology, Multi-Scale Mechanics (MSM), MESA+, \\ University of Twente, P.O. Box 217, 7500 AE Enschede, The Netherlands \\ h. cheng@utwente.nl \\ 2 Graduate School of Environmental and Life Science, Okayama University, \\ 3-1-1 Tsushima Naka, Kita-ku, Okayama 700-8530, Japan
}

\begin{abstract}
Uncertainty exists in geomaterials at contact, microstructural, and continuum scales. To develop predictive, robust multi-scale models for geotechnical problems, the new challenge is to allow for the propagation of model/parameter uncertainty (conditioned on laboratory/field measurements) between micro and macro scales. We aim to first quantify these uncertainties using an iterative Bayesian filtering framework. The framework utilizes the recursive Bayes' rule to quantify the evolution of parameter uncertainties over time, and the nonparametric Gaussian mixture model to iteratively resample parameter space. Using the iteratively trained mixture to guide resampling, model evaluations are allocated asymptotically close to posterior modes, thus greatly reducing the computation cost. In this paper, we first respectively quantify the parameter uncertainty of models that are discrete and continuum in nature, namely a discrete particle and an elasto-plastic model. We then link the two models by conditioning their uncertainties on the same stress-strain response, thereby revealing micro-macro parameter correlations and their uncertainties. The micro-macro correlations obtained can be either general for any granular materials that share similar polydispersity or conditioned on the laboratory data of specific ones.
\end{abstract}

Keywords: Uncertainty quantification $\cdot$ Bayesian filtering $\cdot$ Discrete element modeling $\cdot$ Constitutive modeling $\cdot$ Micro-macro parameter correlations

\section{Introduction}

Geomaterials are discontinuous in nature and can behave solid or fluid-like, depending on their internal states and external loads (Jiang and Liu 2014). Recent advances in computing power and material modeling have led to several families of numerical and theoretical models capable of describing complex mechanical behaviors of geomaterials (Guo and Zhao 2016; Li and Dafalias 2012). However, a common issue preventing modern geomechanical models from being practical and robust in engineering applications is the uncertainty associated with material parameters (Hicks et al. 2019). 
In most cases, the ultimate use of these models becomes tedious trial-and-error fitting against experimental data, without considering uncertainty propagation to larger scales.

To model soil constitutive behavior, one could use a bottom-up, namely a micromechanical approach, by either formulating the evolution of force/contact networks based on statistical mechanics or directly simulating individual particles, so that the number of mechanical parameters is kept to the mininum. However, quantifying particle properties can be highly uncertain as the measurements require high precision.

Alternatively, a top-down approach, starting from macroscopic quantities, e.g., stress, strains, energies, etc., can have the same level of predictability as micromechanical approaches (Jefferies and Shuttle 2002; Gao and Zhao 2015), with some parameters directly measurable from in-situ/laboratory experiments. These models are computationally more efficient given their continuum nature. Nevertheless, the number of parameters is large, which makes the model structure (e.g., the interdependence between parameters) unclear, causing great model uncertainty. Moreover, most topdown models have parameters that lack physical explanations. Some tried to justify their model as the macroscopic representation of microscopic states (Jiang and Liu 2016) but a direct link between micro and macro parameters is rarely provided, except for ideal particle systems.

It is possible and in fact appealing to derive continuum models of stress-strain relations, following rigorously formulated evolution laws for microstructures. However, such elegant approaches do not work for realist particle systems, in terms of void ratio, polydispersity, and inertial effects. In this work, we seek a statistical and later a Bayesian approach to link particle and continuum scale models via their parameters selected such that the stress and strain predictions are identical. The idea is to infer parameter correlations for a selected pair of micro and macro models, conditioned on all possible stress-strain behavior of a certain geomaterial like sand. This task is only possible with efficient Bayesian uncertainty quantification and propagation $(\mathrm{UQ}+\mathrm{P})$ or optimization tools that can accomplish the inference with small numbers of model evaluations.

We use an iterative Bayesian filtering framework (Cheng et al. 2019) to quantify parameter uncertainty at both scales. At the particle scale, the discrete element method (DEM) is used to capture the collective behavior of particle assemblies by tracking the kinematics of individual particles. At the continuum scale, we use the NorSand model which is one of classical critical state soil mechanics (CSSM) models available in most commercial software. Bayesian inference and machine learning models are respectively employed to quantify and model the probability distribution of parameters, conditioned on some reference data. This conditional probability distribution (posterior) is recursively updated in time. Before a certain level of accuracy is reached, the Bayesian updating process is repeated with samples redrawn from a new proposal distribution.

The remainder of this paper is organized as follows. We first summarize the iterative Bayesian framework in Sect. 2. We then quantify the parameter uncertainty of the DEM and the NorSand models in Sects. 3 and 4, respectively. Within the same framework, we link the two models by conditioning their uncertainties on the same stress-strain response, thereby revealing micro-macro parameter correlations and their uncertainties (Sect. 5). The micro-macro correlations are either kept general or 
conditioned on experimental measurements of a geomaterial. Conclusions are drawn in Sect. 6.

\section{Iterative Bayesian Filtering}

The joint posterior probability distribution function (PDF) of model states and parameters is approximated by sequential Monte Carlo methods (van Leeuwen 2010) which are well suited for time-dependent inverse problems. To efficiently approximate the posterior PDF, we first sample a parameter space uniformly using quasi-random numbers. In iterative Bayesian filtering, new samples are drawn from a non-parametric Gaussian mixture model trained with posterior probabilities from the previous iteration. The algorithm implemented in an open-source code GrainLearning (Cheng et al. 2019) allows iteratively sampling near potential posterior modes until posterior expectations converge. Here, only key ideas and variables will be introduced for brevity.

Bayesian filtering aims to estimate the augmented state $\hat{\mathrm{x}}_{\mathrm{t}}=\left(\mathrm{x}_{t}, \Theta_{t}\right)$ of model predictions $\mathrm{x}_{t}$ and parameters $\Theta_{t}$ at time $t$, conditioned on a history of reference data $y_{1: t}$, namely the posterior distribution $p\left(\hat{x}_{t} \mid y_{1: t}\right)$. For geomechanical applications, $\mathrm{x}_{t}$ is the macroscopic response of some predictive models. $\Theta_{t}$ denotes the unknown parameters. The data sequence $y_{1: t}$ can be either experimental or numerical. Following sequential importance sampling, $p\left(\hat{x}_{t} \mid y_{1: t}\right)$ is approximated by the importance weights $\left\{w_{t}^{(i)} ; i=1, \ldots, N_{p}\right\}$ evaluated on an ensemble of sampled states $\left(x_{t}^{(i)}, \Theta^{(i)}\right)$, where $\Theta^{(i)}$ is drawn from a proposal distribution. The ensemble and their weights approximate the posterior expectations and variances of any model predictions $f_{t}$ as a function of $\hat{\boldsymbol{x}}_{t}$

$$
\begin{gathered}
\hat{\mathrm{E}}\left[f_{t}\left(\hat{\boldsymbol{x}}_{t}\right) \mid \boldsymbol{y}_{1: t}\right]=\sum_{i=1}^{N_{p}} w_{t}^{(i)} f_{t}\left(\hat{\boldsymbol{x}}_{t}^{(i)}\right), \\
\widehat{\operatorname{Var}}\left[f_{t}\left(\hat{\boldsymbol{x}}_{t}\right) \mid \boldsymbol{y}_{1: t}\right]=\sum_{i=1}^{N_{p}} w_{t}^{(i)}\left(f_{t}\left(\hat{\boldsymbol{x}}_{t}^{(i)}\right)-\hat{\mathrm{E}}\left[f_{t}\left(\hat{\boldsymbol{x}}_{t}\right) \mid \boldsymbol{y}_{1: t}\right]\right)^{2} .
\end{gathered}
$$

A known issue with sequential Monte Carlo methods is weight degeneracy, i.e., all weights become zero except for one. GrainLearning circumvents this issue by reinitializing the ensemble (and weights) with new parameter samples drawn from a proposal density and then continuum Bayesian updating again in time. In this work, only the first iteration uses a non-informative proposal. Samples in all iterations afterward are drawn from the proposal density, i.e., the nonparametric Gaussian mixture trained with weights that approximate the previous posterior PDF $p_{k-1}\left(\boldsymbol{\Theta} \mid \boldsymbol{y}_{1: \boldsymbol{T}}\right)$. Figure 1 shows the training of the Gaussian mixture model and the resampling scheme. 


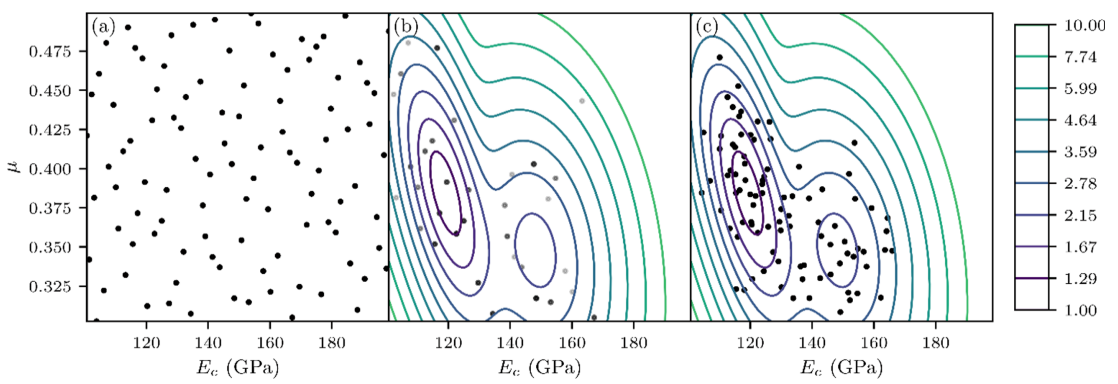

Fig. 1. (a) Initial samples, (b) A mixture model trained with weights on the samples, (c) resampling.

\section{Uncertainty Quantification for the DEM Model}

DEM represents geomaterials as packings of solid particles with simplified geometries and vanishingly small interparticle overlaps. Governed by springs, dashpots, and sliders upon collision, the kinematics of the particles are updated within the explicit time integration scheme, based upon the net forces and moments resulting from particle interactions. To mimic the effect of surface roughness, rolling/twisting stiffness is typically adopted in addition to normal and tangential stiffnesses. Both interparticle tangential forces and contact moments are bounded by Coulomb type yield criteria.

Here, the Hertz-Mindlin contact law derived from the elasticity of two contacting spheres is used. Five micro parameters, namely contact-level Young's modulus $E_{c}$ and Poisson's ratio $v_{c}$, rolling stiffness $k_{r}$ and rolling and sliding friction $\mu_{r}$, and $\mu_{s}$, are kept unknown, because of their relevance to quasi-static responses. Following the random packing generation procedure in (Cheng et al. 2018), the particle assembly in periodic boundary condition was created almost stress-free (null overlaps), which results in a maximum void ratio $e_{\max } \approx 0.68$. Note that $E_{c}$ is very high at this stage in order to ensure very small overlaps during the packing generation stage.

For the DEM model, $\Theta=\left\{E_{c}, v_{c}, k_{r}, \mu_{r}, \mu_{s}\right\}$ and the model prediction $\boldsymbol{x}$ consists of the ratio of deviatoric to mean stress $q / p$ and the volumetric strain $\varepsilon_{v}$, comparable to their counterparts in $\boldsymbol{y}$ measured from drained triaxial compression experiments on Toyoura sand. The axial strain $\varepsilon_{a}$ is not included because both experiments and simulations are controlled by $\varepsilon_{a}$. We consider three confining pressures $\sigma_{c}=0.5,1.0$, and 2.0 MPa to examine the stress dependence of the DEM model. Therefore, both $\boldsymbol{x}_{\boldsymbol{t}}$ and $\boldsymbol{y}_{\boldsymbol{t}}$ in total have 6 elements at time $t$, i.e., $q / p$ and $\varepsilon_{v}$ for each confining pressure.

GrainLearning provides not only Bayesian UQ for the parameters but the ensemble prediction for macroscopic responses as well (ensemble predictions are probabilistic, with weighted averages and standard deviations). The ensemble predictions of $q / p$ and $\varepsilon_{v}$ and the experimental data are compared in Fig. 2. The shaded area shows the model uncertainty resulting from a normalized covariance parameter of 0.1 . It appears the agreement between numerical and experimental results becomes worse as $\sigma_{c}$ increases. This is because particle crushing, to some extent, occurred in the experiments but could not be modeled in the simulations. It is worth noting that all micro parameters are found 
to be independent with each other (see Fig. 9 in Cheng et al. 2018). $E_{c}$ and $\mu_{s}$ are slightly correlated, because of their joint influence on the microstructure.
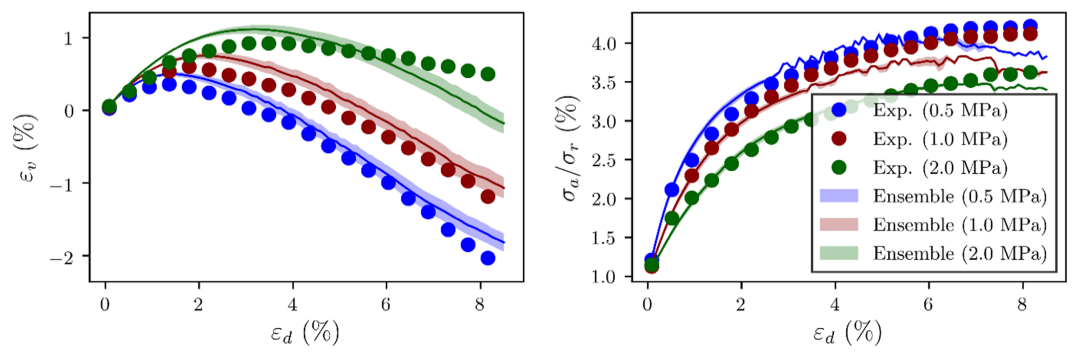

Fig. 2. Comparison of ensemble predictions of the DEM model and experimental result

\section{Uncertain Quantification for the NorSand Model}

The NorSand model is one of the first CSSM models that use the state parameter $\psi=e-e_{c}$ to capture the effect of void ratio and/or confining pressure on the constitutive behavior of particulate materials (Jefferies and Shuttle 2002). Starting from the stress-dilatancy rule $D^{p}=(M-\eta) /(1-N)$, NorSand introduces the concept of the image pressure and its maximum $p_{i}$ and $p_{i, \max }$. The latter evolves with the current $p$ and $\psi$, and defines a bounding surface to which the yield surface expands or shrink, depending on whether a limiting dilatancy is reached. The role of $p_{i}$ is like $p_{c}$ in the Cam-clay model but it allows for predicting softening and dilatancy in conjunction with $\psi$. Most NorSand parameters can be determined from triaxial tests. However, in this work, we set all parameters free to first examine the model structure from the perspective of parameter interdependence. We summarize eight unknown parameters below in Table 1, together with the fixed ones ( $b$ and $\xi$ ) determined directly from our DEM data.

Table 1. NorSand parameters categorized in terms of elasticity, plasticity, and critical state.

\begin{tabular}{l|l|l}
\hline Elasticity $\left(G=G_{0} p^{b}\right)$ & Critical state line $\left(e_{c}=\Gamma-\left(\lambda / p_{a}\right)^{\xi}\right)$ & Plasticity \\
\hline$G_{0}$ & $\Gamma, \lambda, M_{t c}$ & $\chi, N, H_{0}, H_{a}$ \\
\hline
\end{tabular}

We choose to condition the UQ for NorSand on the DEM data created with $E_{c}=$ $8.3 \mathrm{e} 9, v_{c}=0.04, k_{r}=0.358, \mu_{r}=0.476$ and $\mu_{s}=25$ because we aim to (1) compare the capability of NorSand with DEM, and (2) identify micro-macro parameter correlations conditioned on the same macroscopic response. Therefore, like in Sect. 3, $\boldsymbol{x}_{\boldsymbol{t}}$ for NorSand has 6 elements at time $t$, i.e., $q / p$ and $\varepsilon_{v}$ for three confining pressures. One of the DEM model evaluations from Sect. 3 is utilized as $\boldsymbol{y}_{t}$ for NorSand UQ. Except for $\boldsymbol{x}_{\boldsymbol{t}}, \boldsymbol{y}_{t}$ and the parameters, everything else is the same as in Sect. 3 . We test the ability of our iterative Bayesian filtering in high dimensionalities which was not possible with DEM, due to the large computational cost. 

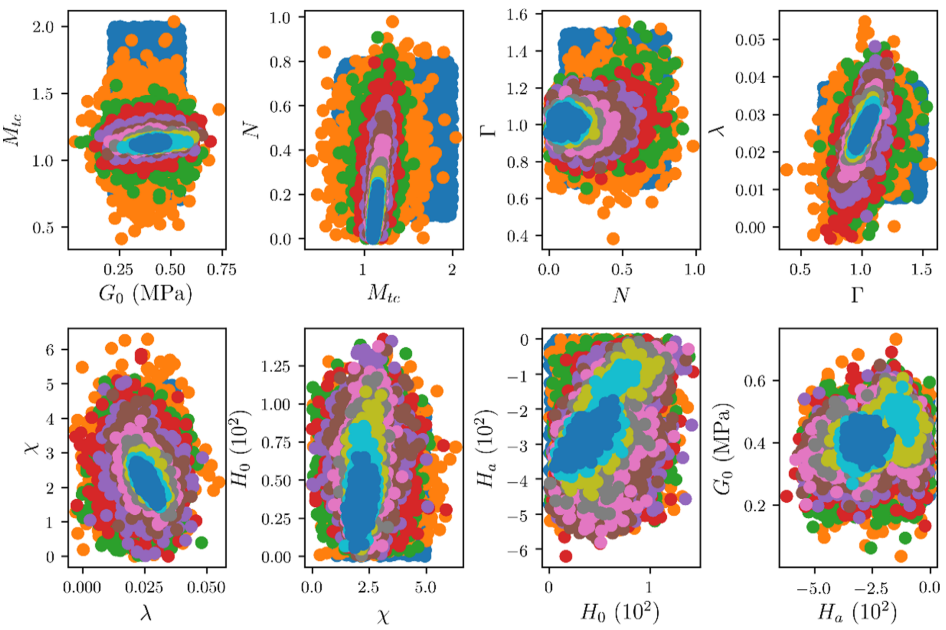

Fig. 3. NorSand parameters resampled at various iterations of Bayesian filtering.
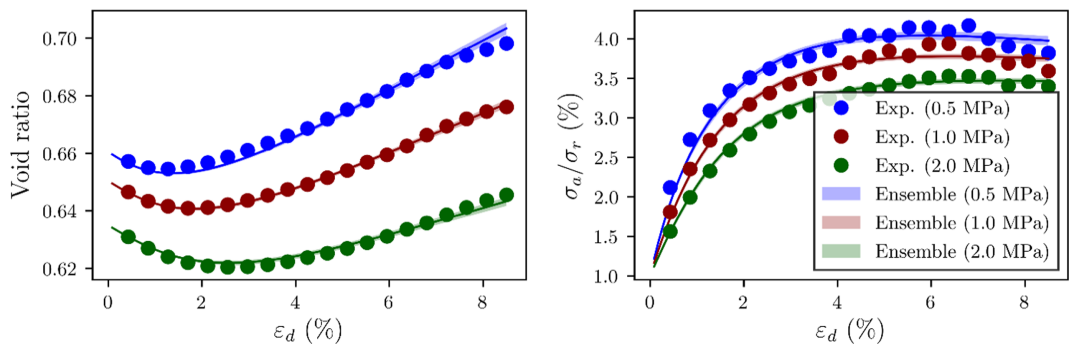

Fig. 4. Comparison of ensemble predictions of the NorSand model and experimental result

The iterations are continued until the normalized covariance parameter is smaller than 0.01. Figure 3 shows the parameter samples converging to some trends, either correlated (e.g., $H_{0}$ and $\left.H_{a}\right)$ or uncorrelated $\left(M_{t c}, G_{0}\right)$, as the number of iterations increases. These (in)dependencies are characterized by the Gaussian mixture model and utilized for resampling. One of the most significant findings is the uncertainty in a few parameters, e.g., $M_{t c}$, is very low, which means they are unique parametrizations of this particulate material. On the contrary, the uncertainty in e.g., $G_{0}$ is large, which is not surprising because of its secondary role in hardening and softening. Evidently, most of the parameters are not uniquely identifiable, e.g., $H_{0}$ and $H_{a}$ highly correlated; there is room for reducing dimensionality and further simplification of the model. Figure 4 shows the ensemble prediction from NorSand in good agreement with the DEM data. 


\section{Uncertainty Propagation Between Micro and Macro Parameters}

Sections 3 and 4 respectively demonstrate the ability of GrainLearning in quantifying parameter uncertainties in geomechanical models at two different scales. The two models are equally accurate in predicting sand-like behavior. Now the question arises as to how the DEM (micro) parameters and the NorSand (macro) parameters are linked, either in a general, unbiased sense or specifically for a certain geomaterial.

Our approach to obtaining general, unbiased micro-macro parameter correlations is to repeat the UQ for NorSand as in Sect. 4 using DEM data generated from quasirandom micro-parameters, i.e., $\log _{10} E_{c} \in[7,10], v_{c} \in[0,0.5], k_{r} \in[0,1], \mu_{r} \in[0,1]$
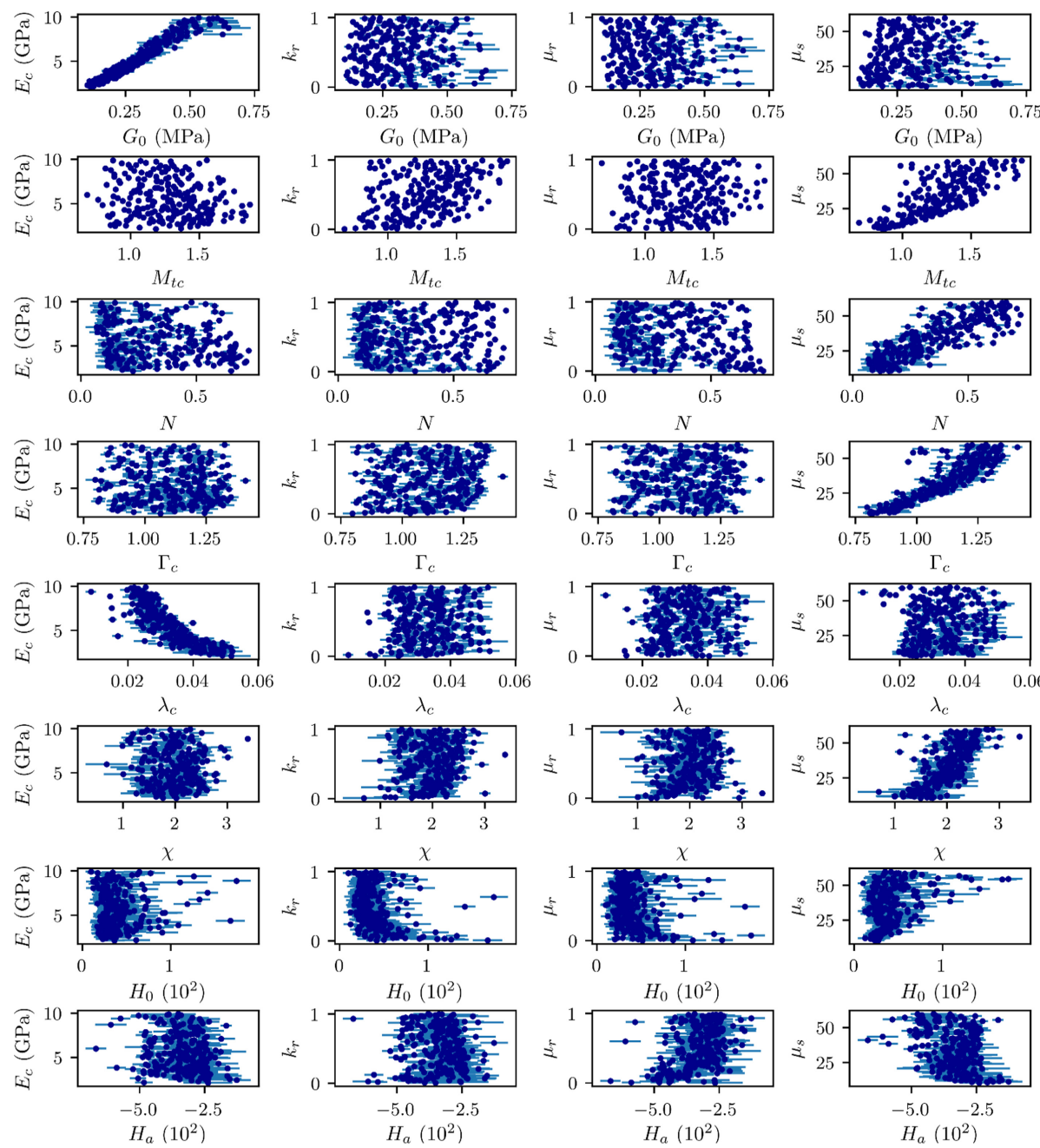

Fig. 5. Correlations between macro and micro parameters (circles) and their uncertainty (error bars). 
and $\mu_{s} \in[10,60]$. A total number of 714 DEM simulations of triaxial compression $\left(\sigma_{c}=0.5,1.0\right.$ and $\left.2.0 \mathrm{MPa}\right)$ as in Sect. 3 were performed. We then repeat the UQ for NorSand against each set of DEM data, keeping the normalized covariance parameter smaller than 0.01. The resulting micro-macro correlations are shown in Fig. 5, with circles and error bars representing the posterior expectations and variances, respectively. The correlations with $v_{c}$ are omitted because of their lesser significance.

Figure 5 clearly shows that $G_{0}$ is almost proportional to $E_{c}$. For the critical state parameters, $M_{t c}$ is uniquely identifiable (invisible error bars) and has a strong correlation with $k_{r}$ and $\mu_{s} ; \Gamma$ and $\lambda$ are dominated by $\mu_{s}$ and $E_{c}$ respectively. For the plasticity parameters, $N$ and $\chi$ seems to be governed by $\mu_{s}$ only, whereas the plastic hardening coefficients $H_{0}$ and $H_{a}$ are collectively controlled by $k_{r}, \mu_{r}$ and $\mu_{s}$.

Although not shown here, it is straight forward to condition the micro-macro correlations and their uncertainties on experimental data, through the importance weights on the DEM data. The micro-macro uncertainty map will degenerate into the posterior distribution that corresponds to the uncertainty in the experimental data.

\section{Conclusions}

In this work, we demonstrate the capability of iterative Bayesian filtering in uncertainty quantification for a discrete particle (DEM) and an elasto-plastic (NorSand) model. By training nonparametric Gaussian mixtures iteratively with statistics from the previous iteration, the (re)sampling algorithm allows sampling asymptotically close to the modes of the probability distribution of model parameters, conditioned on reference data. By assuming a normalized variance in the reference data, parameter uncertainties are efficiently quantified for both models. From the correlation structures, we observe that the micro parameters are mostly independent of each other, whereas some macromacro parameters are strongly correlated, especially the plasticity ones.

Because of the efficiency and robustness of our Bayesian tool, we could massively apply the same technique to find the NorSand parameters (including their uncertainty) which reproduce an ensemble of DEM data, created from quasi-randomly sampled DEM parameters. Future work involves (1) principal component analyses of the micromacro parameter correlation to reduce dimensionality, (2) selecting plausible pairs of micro and macro models that have the same precision, and (3) deploying them in multiscale simulations such that the material behavior is consistent in the two-model coupled overlapping domain.

\section{References}

Cheng, H., Shuku, T., Thoeni, K., Tempone, P., Luding, S., Magnanimo, V.: An iterative Bayesian filtering framework for fast and automated calibration of DEM models. Comput. Methods Appl. Mech. Eng. 350, 268-294 (2019)

Cheng, H., Shuku, T., Thoeni, K., Yamamoto, H.: Probabilistic calibration of discrete element simulations using the sequential quasi-Monte Carlo filter. Granular Matter 20(1), 1-19 (2018). https://doi.org/10.1007/s10035-017-0781-y 
Gao, Z., Zhao, J.: Constitutive modeling of anisotropic sand behavior in monotonic and cyclic loading. J. Eng. Mech. ASCE 142(1), 04015056 (2015)

Guo, N., Zhao, J.: Parallel hierarchical multiscale modelling of hydro-mechanical problems for saturated granular soils. Comput. Methods Appl. Mech. Eng. 305, 37-61 (2016). https://doi. org/10.1016/j.cma.2016.03.004

Hicks, M.A., Varkey, D., van den Eijnden, A.P., de Gast, T., Vardon, P.J.: On characteristic values and the reliability-based assessment of dykes. Georisk Assess. Manage. Risk Eng. Syst. Geohazards 13(4), 313-319 (2019)

Jefferies, M.G., Shuttle, D.A.: Dilatancy in general Cambridge-type models. Geotechnique 52(9), 625-638 (2002). https://doi.org/10.1680/geot.2002.52.9.625

Jiang, Y., Liu, M.: Granular solid hydrodynamics (GSH): a broad-ranged macroscopic theory of granular media. Acta Mech. 225(8), 2363-2384 (2014)

Jiang, Y., Liu, M.: Similarities between GSH, Hypoplasticity and KCR. Acta Geotech. 11(3), 519-537 (2016)

Li, X.S., Dafalias, Y.F.: Anisotropic critical state theory: role of fabric. J. Eng. Mech. 138(3), 263-275 (2012)

van Leeuwen, P.J.: Nonlinear data assimilation in geosciences: an extremely efficient particle filter. Q. J. Roy. Meteorol. Soc. 136(653), 1991-1999 (2010) 